\title{
Hospital Readmissions in Patients With Carbapenem-Resistant Klebsiella pneumoniae
}

\author{
Julia A. Messina, MD, MSc; ${ }^{1,2}$ Eric Cober, MD; ${ }^{3}$ Sandra S. Richter, MD; ${ }^{4}$ Federico Perez, MD; $;{ }^{5,6}$ Robert A. Salata, MD; ${ }^{6}$ \\ Robert C. Kalayjian, MD; ${ }^{7}$ Richard R. Watkins, MD; $;^{8,9}$ Nikole M. Scalera, MD; ${ }^{10}$ Yohei Doi, MD, PhD; ${ }^{11}$ Keith S. Kaye, MD; ${ }^{12}$ \\ Scott Evans, PhD; ${ }^{13}$ Robert A. Bonomo, MD; ${ }^{5,6,14,15}$ Vance G. Fowler Jr, MD, MHS; ${ }^{1,2}$ David van Duin, MD, PhD ${ }^{16}$ \\ for the Antibacterial Resistance Leadership Group
}

\begin{abstract}
BACKGROUND. Various transmission routes contribute to spread of carbapenem-resistant Klebsiella pneumoniae (CRKP) in hospitalized patients. Patients with readmissions during which CRKP is again isolated ("CRKP readmission") potentially contribute to transmission of CRKP.
\end{abstract}

ов jective. To evaluate CRKP readmissions in the Consortium on Resistance against Carbapenems in K. pneumoniae (CRaCKLe).

DESIGN. Cohort study from December 24, 2011, through July 1, 2013.

Setting. Multicenter consortium of acute care hospitals in the Great Lakes region.

PATIENTS. All patients who were discharged alive during the study period were included. Each patient was included only once at the time of the first CRKP-positive culture.

METHods. All readmissions within 90 days of discharge from the index hospitalization during which CRKP was again found were analyzed. Risk factors for CRKP readmission were evaluated in multivariable models.

RESULTS. Fifty-six (20\%) of 287 patients who were discharged alive had a CRKP readmission. History of malignancy was associated with CRKP readmission (adjusted odds ratio [adjusted OR], 3.00 [95\% CI, 1.32-6.65], $P<.01$ ). During the index hospitalization, 160 patients $(56 \%)$ received antibiotic treatment against CRKP; the choice of regimen was associated with CRKP readmission $(P=.02)$. Receipt of tigecycline-based therapy (adjusted OR, 5.13 [95\% CI, 1.72-17.44], using aminoglycoside-based therapy as a reference in those treated with anti-CRKP antibiotics) was associated with CRKP readmission.

CONCLUSION. Hospitalized patients with CRKP_-specifically those with a history of malignancy-are at high risk of readmission with recurrent CRKP infection or colonization. Treatment during the index hospitalization with a tigecycline-based regimen increases this risk.

Infect. Control Hosp. Epidemiol. 2016;37(3):281-288

In spite of aggressive treatment, carbapenem-resistant Klebsiella pneumoniae (CRKP) infections remain associated with high morbidity and mortality. ${ }^{1,2}$ Posing a global threat, CRKP are now endemic in areas worldwide including in the United States, Asia, India, Europe, and South America. ${ }^{3}$ Klebsiella spp. expressing $K$. pneumoniae carbapenemases are the most common carbapenem-resistant Enterobacteriaceae (CRE) in the United States and have increased rapidly in prevalence during the past 2 decades. ${ }^{4,5}$

The rising prevalence of CRKP impacts infection control policies within healthcare settings. K. pneumoniae carbapenemase $\beta$-lactamases encoded by $b l a_{\mathrm{KPC}}$ may be acquired through both

Affiliations: 1. Division of Infectious Diseases, Duke University, Durham, North Carolina; 2. Duke Clinical Research Institute, Duke University, Durham, North Carolina; 3. Department of Infectious Diseases, Cleveland Clinic, Cleveland, Ohio; 4. Department of Laboratory Medicine, Cleveland Clinic, Cleveland, Ohio; 5. Research Service, Louis Stokes Veterans Affairs Medical Center, Cleveland, Ohio; 6. Division of Infectious Diseases and HIV Medicine, Department of Medicine, Case Western Reserve University School of Medicine, Cleveland, Ohio; 7. Department of Medicine, MetroHealth Medical Center, Cleveland, Ohio; 8. Department of Internal Medicine, Northeast Ohio Medical University, Rootstown, Ohio; 9. Division of Infectious Diseases, Akron General Medical Center, Akron, Ohio; 10. Division of Infectious Diseases, Summa Health System, Akron, Ohio; 11. Division of Infectious Diseases, University of Pittsburgh School of Medicine, Pittsburgh, Pennsylvania; 12. Division of Infectious Diseases, Detroit Medical Center, Wayne State University, Detroit, Michigan; 13. Department of Biostatistics and the Center for Biostatistics in AIDS Research, Harvard School of Public Health, Boston, Massachusetts; 14. Department of Molecular Biology and Microbiology, Case Western Reserve University School of Medicine, Cleveland, Ohio; 15. Department of Pharmacology, Case Western Reserve University School of Medicine, Cleveland, Ohio; 16. Division of Infectious Diseases, University of North Carolina, Chapel Hill, North Carolina.

Presented in part: IDWeek 2014; Philadelphia, Pennsylvania; October 11, 2014 (abstract 1804). The contents are solely the responsibility of the authors and do not necessarily represent the official views of the National Institutes of Health.

Received September 4, 2015; accepted November 4, 2015; electronically published December 21, 2015

(c) 2015 by The Society for Healthcare Epidemiology of America. All rights reserved. 0899-823X/2016/3703-0005. DOI: 10.1017/ice.2015.298 
clonal and plasmid expansion, facilitating spread of carbapenem resistance among Enterobacteriaceae. ${ }^{6}$ Various routes of transmission were demonstrated in recent CRKP outbreaks. ${ }^{7-9}$ Patients residing in long-term care facilities who are subsequently admitted to acute care hospitals are thought to significantly contribute to the transmission of CRKP. A recent study confirms that patients who are admitted to acute care hospitals from highacuity long-term care facilities are more likely to be colonized with K. pneumoniae carbapenemase-producing Enterobacteriaceae. ${ }^{10}$

As hospital readmissions of patients with persistent or recurrent CRKP may contribute to the accelerated spread of this pathogen, rates of and risk factors for readmission in patients with CRKP during which the organism is again isolated are important to delineate. In order to better understand the manner in which CRKP is disseminated in the Great Lakes region, we sought to determine how often patients infected or colonized with CRKP were readmitted with repeat positive cultures for CRKP and whether the choice of treatment regimen directed against CRKP influenced CRKP readmission rates.

\section{METHODS}

\section{Design}

A nested cohort study was conducted within the Consortium on Resistance against Carbapenems in Klebsiella pneumoniae (CRaCKle) cohort, which was previously described. ${ }^{11}$ Briefly, CRaCKle is a multicenter, prospective, longitudinal, observational study of hospitalized patients with positive cultures for CRKP in the Great Lakes region. The cohort consists of CRaCKle patients who survived their index hospitalization and whose index hospitalization started after December 24, 2011, and ended on or before July 1, 2013. Routine screening of asymptomatic patients for CRKP carriage was not performed at any of the study sites during the study period. The institutional review boards of all sites involved approved the study.

\section{Definitions}

The primary outcome of this study was CRKP readmission, which was defined as a hospital readmission within 90 days of the index hospitalization during which CRKP was again cultured from the patient. The index hospitalization was defined as the first hospitalization within the study period during which CRKP was identified. Each patient was included only once at the time of the index hospitalization.

Standardized definitions of infection were used, as previously described. ${ }^{11}$ Treatment regimens effective against CRKP were defined as follows: receipt of an aminoglycoside, colistin, tigecycline, trimethoprim-sulfamethoxazole, or fosfomycin unless in vitro resistance was documented to that antimicrobial in the patient's isolate. In all instances guidelines from the Clinical and Laboratory Standards Institute (aminoglycosides, trimethoprim-sulfamethoxazole, and fosfomycin) and the European Committee on Antimicrobial Susceptibility Testing (colistin and tigecycline) were followed. For analysis purposes, the type of regimen was assigned as previously reported. ${ }^{12}$ Briefly, any regimen that contained an aminoglycoside was deemed "aminoglycoside-based," then any regimen that contained colistin but not an aminoglycoside was designated "colistin-based," then any regimen that contained tigecycline but not colistin or aminoglycoside was regarded as "tigecyclinebased." All other regimens were classified as "other." Chronic kidney disease was defined as a serum creatinine level greater than $2 \mathrm{mg} / \mathrm{dL}$ upon admission. Critical illness was designated using a Pitt bacteremia score greater than or equal to 4 points on the day of the index culture. ${ }^{13}$ The Charlson Comorbidity Index was calculated as described. ${ }^{14}$

\section{Microbiology}

In our study CRKP are K. pneumoniae isolates with nonsusceptibility per Clinical and Laboratory Standards Institute guidelines to the following carbapenems: meropenem, imipenem, or ertapenem..$^{15}$ Bacterial identification and routine antimicrobial susceptibility testing were performed with MicroScan (Siemens Healthcare Diagnostics) or Vitek2 (bioMérieux), supplemented by GN4F Sensititre tray (Thermo Fisher) or Etest (bioMérieux), as indicated. In more than $90 \%$ of tested isolates, carbapenem resistance was mediated through $b l a_{\mathrm{KPC}-2}$ or $b l a_{\mathrm{KPC}-3}$, as previously described. ${ }^{11}$

\section{Statistical Analysis}

Differences between groups were analyzed using the Wilcoxon rank sum test for continuous variables. The Fisher exact and Pearson tests were used for categorical variables where appropriate. All variables that were associated with CRKP readmission at the $P<.1$ level were included in multivariable logistic models, and adjusted ORs with associated confidence intervals were calculated. A Kaplan-Meier curve was constructed to compare time to readmission. A Cox proportional hazards model on time to 90-day readmission was used to calculate adjusted hazard ratios. All variables that were associated with CRKP readmission at the $P<.1$ level were included in this model in addition to treatment variables. $P$ values of $\leq .05$ were considered statistically significant. JMP software, version 10.0.1 (SAS), was used for all analyses.

\section{RES ULTS}

\section{Patients}

The demographic characteristics of the 287 patients who met inclusion criteria are summarized in Table 1. CRKP infection was present during index hospitalization in 109 patients (38\%), and the remaining 178 patients were classified as having CRKP colonization. During the index hospitalization, 192 patients $(67 \%)$ had CRKP isolated from a urine sample, 32 (11\%) had CRKP isolated from respiratory specimens, 30 (10\%) had CRKP isolated from wounds, 24 (8\%) had CRKP isolated from blood, and 9 (3\%) had CRKP isolated from 
т A в LE 1. Clinical Characteristics in Study of Hospital Readmissions in Patients With CRKP

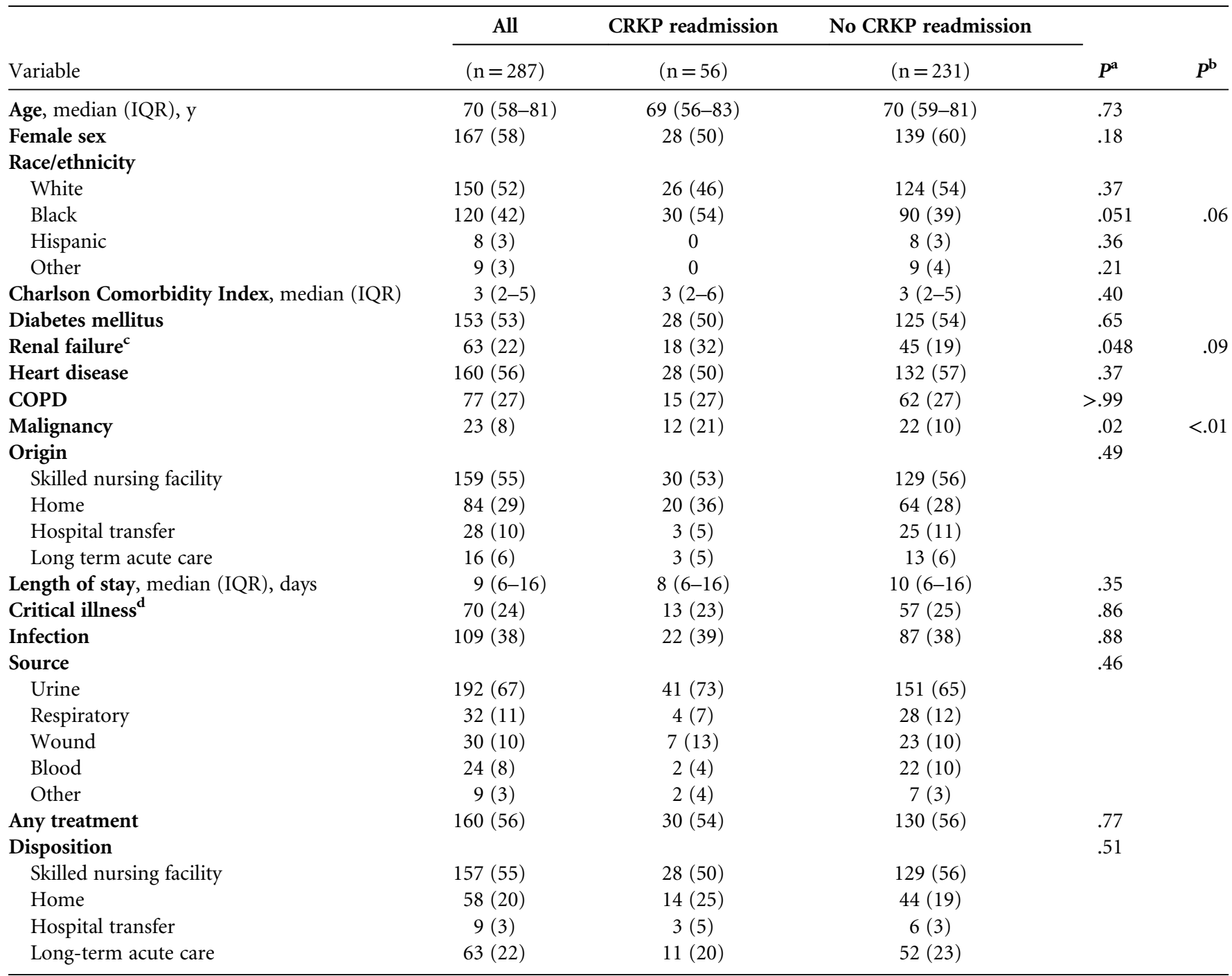

NOTE. All data are expressed as n (\%), unless otherwise indicated. CRKP, carbapenem-resistant Klebsiella pneumoniae; COPD, chronic obstructive pulmonary disease; IQR, interquartile range; LOS, length of stay.

${ }^{\mathrm{a}}$ Univariable relationship between variable of interest and CRKP readmission.

${ }^{\mathrm{b}}$ Multivariable model including black race, renal failure, and malignancy.

${ }^{c}$ Renal failure defined as creatinine $>2 \mathrm{mg} / \mathrm{dL}$ upon admission.

${ }^{\mathrm{d}}$ Critical illness defined as Pitt bacteremia score $\geq 4$ at the time of index culture.

"other" sites, which included abdominal sources such as bile, ascites, and abdominal abscess.

Fifty-six (20\%) of 287 patients had a readmission during which CRKP was again isolated ("CRKP readmission") within 90 days. We evaluated 17 patients for whom we had paired isolates from index admission and readmission. In 16 (94\%) of the 17 patients the same repetitive sequence-based polymerase chain reaction strain was identified upon readmission. During their readmission, 22 patients (39\%) had CRKP infection while 34 (61\%) had colonization of a site with CRKP (Table 1). In univariable analysis, chronic kidney disease and history of malignancy were significantly associated with CRKP readmission within 90 days. Eighteen $(32 \%)$ of 56 patients with CRKP readmission had chronic kidney disease compared with 45 (19\%) of 231 patients without CRKP readmission $(P=.048)$. A history of malignant tumor was present in $12(21 \%)$ of 56 patients with CRKP readmission, compared with $22(10 \%)$ of 231 other patients $(P=.02)$. In addition, a trend toward increased CRKP readmissions was seen in black patients; 30 (54\%) of 56 patients with CRKP readmission were black compared with 90 (39\%) of 231 patients without CRKP readmission $(P=.051)$. In a multivariable model that included chronic kidney disease, history of malignancy, and black race, only a history of malignancy remained associated with CRKP readmission (OR, 3.00 [95\% CI, 1.32-6.65], $P<.01)$. Sex, age, and CRKP colonization vs infection status were not associated with CRKP readmission. 
In addition, a trend was seen toward more CRKP readmissions in patients with index isolates resistant to trimethoprimsulfamethoxazole $(P=.07)$. Other antimicrobial susceptibility testing results were also not significantly associated with CRKP readmission (Table 2).

\section{Treatment}

During the index hospitalization, 160 patients $(56 \%)$ received antibiotics directed against CRKP within the first 7 days of the first positive culture for CRKP. Being treated with antibiotics with in vitro activity against CRKP was not associated with CRKP readmission; 30 (54\%) of the 56 patients with CRKP readmissions received some form of treatment, whereas $130(56 \%)$ of the 231 without CRKP readmissions were treated $(P=.77)$.

The impact of specific treatment choices on CRKP readmissions was then evaluated in patients who received antibiotics effective against CRKP during their index hospitalization (Table 3). In univariable analysis, patients who received more than 1 drug with in vitro activity against CRKP were more likely to have a CRKP readmission; 13 (31\%) of 42 patients treated with more than 1 drug were readmitted vs $17(14 \%)$ of 118 patients treated with a single agent $(\mathrm{OR}, 2.66$ [95\% CI, 1.16-6.12], $P=.02)$.

TABLE 2. Antimicrobial Susceptibilities in Study of Hospital Readmissions in Patients With CRKP

\begin{tabular}{|c|c|c|c|}
\hline Variable & All & CRKP readmission & No CRKP readmission \\
\hline No. of patients & 287 & $56(20)$ & $231(80)$ \\
\hline \multicolumn{4}{|c|}{ Amikacin $^{\mathrm{a}}$} \\
\hline Not tested & 89 & $15(17)$ & $74(83)$ \\
\hline Susceptible & 151 & $31(21)$ & $120(79)$ \\
\hline Intermediate & 10 & 0 & $10(100)$ \\
\hline Resistant & 37 & $10(27)$ & $27(73)$ \\
\hline \multicolumn{4}{|l|}{ Gentamicin $^{\mathrm{a}}$} \\
\hline Not tested & 2 & 0 & $2(100)$ \\
\hline Susceptible & 114 & $22(19)$ & $92(81)$ \\
\hline Intermediate & 25 & $6(24)$ & $19(76)$ \\
\hline Resistant & 146 & $28(19)$ & $118(81)$ \\
\hline \multicolumn{4}{|l|}{ Colistin ${ }^{\mathrm{b}}$} \\
\hline Not tested & 160 & $30(19)$ & $130(81)$ \\
\hline Susceptible & 117 & $25(21)$ & $92(79)$ \\
\hline Resistant & 10 & $1(10)$ & $9(90)$ \\
\hline \multicolumn{4}{|l|}{ Tigecycline $^{\mathrm{b}}$} \\
\hline Not tested & 84 & $16(19)$ & $68(81)$ \\
\hline Susceptible & 107 & $25(23)$ & $82(77)$ \\
\hline Intermediate & 60 & $10(17)$ & $50(83)$ \\
\hline Resistant & 36 & $5(14)$ & $31(86)$ \\
\hline \multicolumn{4}{|l|}{ TMP/SMX ${ }^{\mathrm{a}}$} \\
\hline Not tested & 9 & 0 & $9(100)$ \\
\hline Susceptible & 82 & $11(13)$ & $71(87)$ \\
\hline Resistant & 196 & $45(23)$ & $151(77)$ \\
\hline
\end{tabular}

NOTE. All data are expressed as n (\%), unless otherwise indicated. CRKP, carbapenem-resistant Klebsiella pneumoniae; TMP-SMX, trimethoprim-sulfamethoxazole.

${ }^{a}$ Based on Clinical and Laboratory Standards Institute guidelines.

${ }^{\mathrm{b}}$ Based on European Committee on Antimicrobial Susceptibility Testing.
Of the 160 treated patients, most were treated with either an aminoglycoside-based regimen (70 [44\%]) or a tigecycline-based regimen $(49[31 \%])$ during their index hospitalization. Of 30 treated patients with CRKP readmission, 14 (47\%) were treated with a tigecycline-based regimen compared with $35(27 \%)$ of the 130 treated patients without CRKP readmissions (OR, 2.38 [95\% CI, 1.05-5.37], $P=.047$ ). When evaluating the receipt of tigecycline during the index hospitalization-regardless of other anti-CRKP antibiotics-a similar association between tigecycline use and CRKP readmission was observed (OR, 2.64 [95\% CI, 1.15-6.09], $P=.03$ ). Fosfomycin use during the index hospitalization occurred in a total of $17(11 \%)$ of the 160 treated patients and was also associated with CRKP readmission in univariable analysis (OR, 3.65 [95\% CI, 1.26-10.58], $P=.02$ ). In multivariable analysis (Table 4), regimen base remained significantly associated with CRKP readmission $(P=.02)$. Using patients who received aminoglycoside-based therapy as a reference group, the adjusted OR of tigecycline-based therapy was 5.13 (95\% CI, 1.72-17.44). In addition, receipt of more than 1 anti-CRKP antibiotic during index hospitalization was strongly associated with CRKP readmission (adjusted OR, 5.14 [95\% CI, 1.78-16.41], $P<.01)$. When comparing patients who received more than 1 with those who received only 1 anti-CRKP antibiotic, no significant differences were found in age, Charlson Comorbidity Index, or Pitt bacteremia score.

\section{Time to CRKP Readmission}

Tigecycline-based treatment was associated with a decreased time to 90 -day CRKP readmission $(P=.04$ by log-rank, Figure 1A). Similarly, receiving any tigecycline-regardless of receipt of other antibiotics with in vitro activity against CRKP-was associated with decreased time to 90-day CRKP readmission $(P=.02$ by log-rank, Figure 1B). In Cox proportional hazards analysis (Table 5), treatment regimen base remained significantly associated with time to 90-day CRKP readmission $(P=.02)$. Using the patients who received aminoglycoside-based therapy as a reference group, the adjusted hazard ratio for tigecycline-based therapy was 4.33 (95\% CI, 1.67-11.60). Of note, when urinary source was forced into the model as a confounding variable, the association between treatment regimen and time to CRKP readmission remained significant (data not shown). In addition, receipt of more than 1 in vitro active antibiotic in the first 7 days after the first positive CRKP culture during the index hospitalization was also associated with time to 90-day CRKP readmission (adjusted hazard ratio, 4.46 [95\% CI, 1.77-11.36]; $P<.01$ ). To determine whether the association between tigecycline-based therapy and time to 90-day CRKP readmission was dependent on infection status during index hospitalization, a stratified analysis was performed. In both the CRKP colonization group as well as the CRKP infection group, treatment base was associated with time to 90-day CRKP readmission in Cox proportional hazard analysis $(P=.03$ and $P=.04$, respectively). The hazard ratios of tigecycline-based 
TABLE 3. Treatment Characteristics in Study of Hospital Readmissions in Patients With CRKP

\begin{tabular}{lcccc}
\hline Variable & All & $\begin{array}{c}\text { CRKP } \\
\text { readmission }\end{array}$ & $\begin{array}{c}\text { No CRKP } \\
\text { readmission }\end{array}$ & $\boldsymbol{P}$ \\
\hline $\begin{array}{l}\text { No. of patients } \\
\text { Any in vitro active }\end{array}$ & 160 & $30(19)$ & $130(81)$ & \\
$\quad$ drug in first 7 days & & & & \\
$\quad$ Aminoglycoside & $70(44)$ & $11(37)$ & $59(45)$ & .42 \\
$\quad$ Colistin & $27(17)$ & $5(17)$ & $22(17)$ & $>.99$ \\
Tigecycline & $76(48)$ & $20(67)$ & $56(43)$ & .03 \\
TMP-SMX & $14(9)$ & $1(3)$ & $13(10)$ & .47 \\
Fosfomycin & $17(11)$ & $7(23)$ & $10(8)$ & .02 \\
Base of regimen & & & & \\
$\quad$ Aminoglycoside & $70(44)$ & $11(37)$ & $59(45)$ & .42 \\
Colistin & $22(14)$ & $3(10)$ & $19(15)$ & .77 \\
$\quad$ Tigecycline & $49(31)$ & $14(47)$ & $35(27)$ & .047 \\
$\quad$ Other & $19(12)$ & $2(7)$ & $17(13)$ & .53 \\
$>$ 1 in vitro active & $42(26)$ & $13(43)$ & $29(22)$ & .02 \\
$\quad$ drug in first 7 days & & & & \\
\hline
\end{tabular}

NOTE. All data are expressed as n (\%), unless otherwise indicated. CRKP, carbapenem-resistant Klebsiella pneumoniae; TMP-SMX, trimethoprim-sulfamethoxazole.

TABLE 4. Multivariable Logistic Regression for CRKP Readmission in 160 Treated Patients

\begin{tabular}{llll}
\hline Variable & \multicolumn{1}{c}{ OR } & \multicolumn{1}{c}{$\mathbf{9 5 \%}$ CI } & \multicolumn{1}{c}{$\boldsymbol{P}$} \\
\hline Black race & 1.69 & $0.68-4.23$ & .26 \\
History of malignant tumor & 4.07 & $1.14-14.45$ & .03 \\
Renal failure & 1.10 & $0.37-3.05$ & .86 \\
Base of regimen & & & .02 \\
Aminoglycoside & 1 [Reference] & & \\
Colistin & 1.26 & $0.24-5.30$ & \\
Tigecycline & 5.13 & $1.72-17.44$ & \\
Other & 2.03 & $0.26-11.57$ & \\
$>\mathbf{1}$ in vitro active drug in first & 5.14 & $1.78-16.41$ & $<.01$ \\
$\quad \mathbf{7}$ days & & & \\
\hline
\end{tabular}

NOTE. All data are expressed as n (\%), unless otherwise indicated. CRKP, carbapenem-resistant Klebsiella pneumoniae; OR, odds ratio.

therapy, when using aminoglycoside-based therapy as a reference, were 3.99 (95\% CI, 1.20-14.31) and 6.56 (95\% CI, 1.39-34.81) for colonization and infection, respectively.

\section{DISC USSION}

The present study evaluates readmission rates with CRKP and analysis of risk factors from a prospective multicenter cohort. We observed that it was common for hospitalized patients with CRKP infection or colonization to have a readmission during which CRKP was again isolated. Interestingly, this occurred in $20 \%$ of patients who survived their index hospitalization. This finding suggests that patients with CRKP-especially those patients treated with tigecycline and those with a history of malignant tumor-carry CRKP for prolonged periods and have frequent and recurrent healthcare exposures during which they are likely to interact with other vulnerable patients. In contrast, the presence of CRKP infection vs CRKP colonization did not appear to have an impact on CRKP readmission rates.

The observation of CRKP readmission is an outcome that requires 2 related but distinct occurrences. First, the patient needs to be readmitted, and second, during that readmission, CRKP must be cultured from a clinically important site. Thus, in the current study, we evaluated the overlapping risk factors for hospital readmission and prolonged CRKP carriage.

Hospital readmission rates are the subject of multiple studies to identify risk factors for readmission. ${ }^{16,17}$ Hospital reimbursement is increasingly being linked to readmission rates. In a recent report of 90-day readmissions following hospitalization for severe sepsis, the investigators noted a $42.6 \%$ readmission rate, with $41.6 \%$ of these readmissions being for potentially preventable conditions such as heart failure exacerbation, pneumonia, and urinary tract infection. ${ }^{18}$ Potentially preventable readmissions occurred significantly more frequently in patients with severe sepsis compared with matched control subjects with other acute care diagnoses.

During a 90-day period, a high rate of finding CRKP again during readmission was consistent with findings from studies of duration of CRE carriage. In 1 study, among patients who had CRE isolated during their index hospitalization, $78 \%$ of patients still had CRE carriage at 3 months and 39\% still had detectable CRE carriage at 1 year. ${ }^{19}$ Those patients who were readmitted and in whom CRE was isolated in a clinical culture as opposed to a surveillance culture had significantly longer CRE carriage (641 days vs 387 days). ${ }^{19}$ Another study identified risk factors for recurrent positive CRE screens during hospital encounters, including prior fluoroquinolone use, admission from another hospital or healthcare facility, and hospital readmission within 3 months of initial positive CRE screen. ${ }^{20}$ In a case-control study of recurrence of CRE carriage from Israel, recurrence of CRE was common after presumed eradication at 6 months after last positive sample and associated with recurrent admissions after presumed eradication. ${ }^{21}$

The current study did not directly address the role of CRE decolonization as a means to reduce the risk of future infection and spread of CRE to other patients. Decolonization is another potential target for infection control measures in high-risk patients. In experimental models, oral high-dose polymyxin therapy resulted in long-term elimination of CRE carriage. ${ }^{22}$ This principle was then evaluated in a pilot study of selective digestive decontamination for eradication of CRKP carriage. A double-blind, randomized controlled trial was conducted in 40 patients comparing oral gentamicin and polymixin $\mathrm{E}$ vs placebo. ${ }^{23}$ The investigators showed the CRKP isolation in rectal cultures was significantly reduced by 2 weeks, and this reduction was maintained through the 6-week period of the study.

Patients with malignant tumors were found to be at increased risk for CRKP readmission. This is likely secondary to an increased overall readmission risk in this cohort. In addition, 


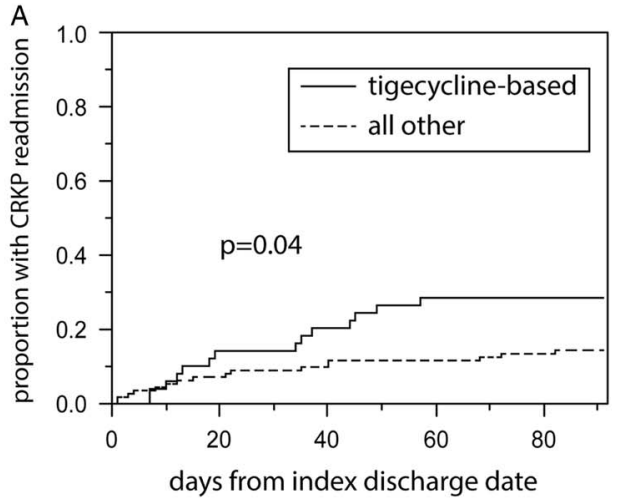

No. at risk:

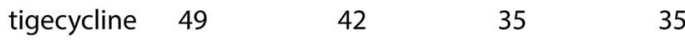
$\begin{array}{lllll}\text { all other } & 111 & 101 & 99 & 95\end{array}$

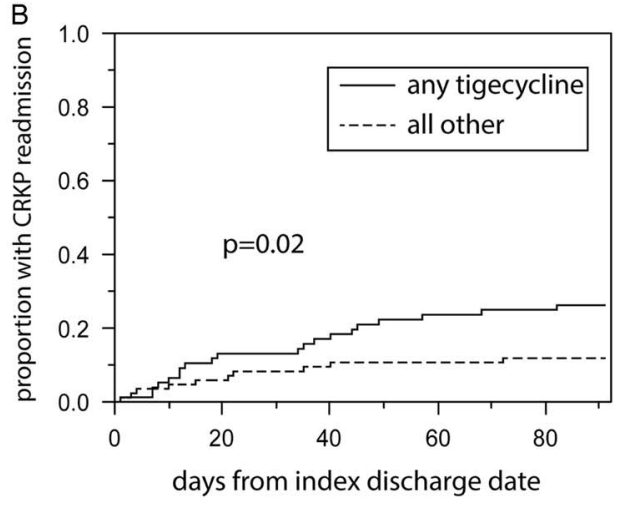

$\begin{array}{llll}76 & 67 & 59 & 57 \\ 84 & 78 & 76 & 75\end{array}$

FIGURE 1. Time-to-90-day carbapenem-resistant Klebsiella pneumoniae (CRKP) readmission for patients who received anti-CRKP antibiotics $(\mathrm{n}=160)$ during their index hospitalization. A, Comparing patients who received tigecycline-based treatment during index hospitalization $(\mathrm{n}=49)$ vs all others $(\mathrm{n}=111) . B$, Comparing patients who received any tigecycline during index hospitalization $(\mathrm{n}=76)$ vs all others $(\mathrm{n}=84)$.

TABLE 5. Cox Proportional Hazards Model on Time to CRKP Readmission Within 90 Days in 160 Treated Patients

\begin{tabular}{|c|c|c|c|}
\hline Variable & aHR & $95 \% \mathrm{CI}$ & $P$ \\
\hline Black race & 1.93 & $0.85-4.30$ & .12 \\
\hline Malignant tumor & 3.17 & $1.12-7.83$ & .03 \\
\hline Renal failure & 1.06 & $0.41-2.46$ & .88 \\
\hline Base of regimen & & & .02 \\
\hline Aminoglycoside & 1 [Reference] & & \\
\hline Colistin & 1.28 & $0.29-4.15$ & \\
\hline Tigecycline & 4.33 & $1.67-11.60$ & \\
\hline Other & 1.87 & $0.26-8.80$ & \\
\hline $\begin{array}{l}>1 \text { in vitro active drug in first } \\
7 \text { days }\end{array}$ & 4.46 & $1.77-11.36$ & $<.01$ \\
\hline
\end{tabular}

NOTE. aHR, adjusted hazard ratio; CRKP, carbapenem-resistant Klebsiella pneumoniae.

cancer has been linked to microbiome changes. ${ }^{24}$ This may theoretically influence the duration of CRKP carriage.

The finding of an increased risk of CRKP readmission when patients are treated with tigecycline raises concern for a potential relationship between tigecycline use and risk for subsequent CRKP treatment failure whether demonstrated through recurrent infection or persistent colonization. A number of potential explanations for this observed association could be considered. Because this is an observational study, our data may simply reflect confounding by indication. For some unmeasured reason, patients who are more likely to receive tigecycline may be the same patients who are more likely to get readmitted and have CRKP again isolated. Alternatively, this may represent a true association. Meta-analyses evaluating randomized controlled trials on the use of tigecycline in non-CRE infections are suggestive of inferior efficacy vs comparators. ${ }^{25-28}$ Second, tigecycline is bacteriostatic rather than bactericidal and has a low urinary excretion. This antibiotic property likely plays an important role in this context; one wonders whether the numbers of CRKP are not reduced sufficiently. Third, current tigecycline dosing strategies (especially monotherapy) may not be optimal for treating multidrug-resistant bacteria such as CRKP. ${ }^{29}$ Lastly, tigecycline was also shown in murine models to promote the intestinal overgrowth of CRKP. ${ }^{22}$ In subgroup analysis, the association between tigecycline treatment and CRKP readmission was observed in both the group of patients with CRKP infection as well as the group with CRKP colonization.

Identifying risk factors for CRE isolation, infection, and readmission can potentially reduce patient-to-patient transmission of CRE within healthcare facilities. Multiple studies have reported successful reduction in rates of epidemic CRE through targeted surveillance and infection control measures. ${ }^{30-33}$ Further study is needed to determine whether infection control measures such as routine culture screening, empirical contact isolation upon readmission, and standardized environmental cleaning protocols implemented in previous studies would be beneficial to patients at risk for CRE readmission.

Within the present study, receipt of more than 1 antibiotic with in vitro activity against CRKP in the first 7 days after a positive culture was also significantly associated with CRKP readmission. Our data cannot explain why this association was observed, but it is possibly related to reasons for adding or changing antibiotic regimens, such as perceived failure of therapy or the occurrence of side effects. Alternatively, the use of several antibiotics may lead to increased disruption of the gut microbiome, which in turn could lead to persistence of CRKP carriage. Similarly, antibiotic usage was linked to recurrence of CRE carriage in the case-control study by Bart et al. ${ }^{21}$

Limitations of this study include its observational nature; patients were not actively screened for CRKP on hospital admission, and antibiotic treatment was not randomized but based on clinical indication. As in all observational studies, we 
can report only on association rather than on causality. However, our study represents an inclusive cohort of consecutive patients admitted at various different hospitals being treated in a way that reflects current medical practice. Additionally, among patients who did not have a 90-day CRKP readmission, data were not collected beyond their index hospitalizations. Nonetheless, if patients were readmitted with CRKP during that time frame it is most likely that this readmission would have happened within the CRaCKle network and would have been captured in our cohort because the consortium covers most area hospitals.

In conclusion, we have established that in our population $20 \%$ of hospitalized patients with CRKP are readmitted within 90 days with repeat isolation from CRKP from clinical cultures. Interestingly, many of these isolates are the same strain as the index isolate. These patients contribute to the CRKP colonization pressure in acute care settings. Furthermore, we found that a history of malignant tumor and choice of treatment impact this risk. Further studies in patients with CRE are needed to better characterize relationships between treatment, subsequent risk for readmission, duration of CRE carriage, and risk for subsequent CRE infection. Moreover, identifying patients at risk for CRKP treatment failure and readmission and intervening through infection control measures and choice of treatment regimens based on mechanism of action should be important future directions arising from this study.

\section{ACKNOWLEDGMENTS}

Financial support. National Institute of Allergy and Infectious Diseases of the National Institutes of Health (award UM1AI104681; and Division of Microbiology and Infectious Diseases protocol 10-0065 and RO1 1R01AI119446-01 to K.S.K.), the Clinical and Translational Science Collaborative of Cleveland (to D.V.D. and F.P.), the National Center for Advancing Translational Sciences component of the National Institutes of Health (UL1TR000439), NIH Roadmap for Medical Research; National Institutes of Health (Mid-Career Mentoring Award K24-AI093969 to V.G.F.; and grants AI072219-05 and AI063517-07 to R.A.B.; and research awards R01AI104895 and R21AI107302 to Y.D.); Veterans Affairs Merit Review Program (to R.A.B.), and the Geriatric Research Education and Clinical Center VISN 10 (to R.A.B.), the Research Program Committees of the Cleveland Clinic (to D.V.D.), Steris (unrestricted research grant to D.V.D.).

Potential conflicts of interest. S.S.R. reports that she has received research support from bioMérieux, BD Diagnostics, BioFire, OpGen, Forest Laboratories, Achaogen, Nanosphere, and Pocared, and an honorarium from bioMérieux. Y.D. reports that he has received grant support from Merck and $\mathrm{NIH}$ and consulting fees from Melinta, and serves on the advisory board of Shionogi. K.S. K. reports that he has contact with Forest Laboratories as paid consultant and as grant investigator, and has received an honorarium from its Speaker's Bureau. R.A.B. reports that he has contact with AstraZeneca as grant investigator and grant recipient, with Merck as grant investigator and grant recipient, with Melinta as grant investigator and grant recipient, with Steris as grant investigator and grant recipient, with $\mathrm{NIH}$ as grant investigator and grant recipient, and with VA Merit Review as grant investigator and grant recipient. V.G.F. reports that he has received grant/ research support from Advanced Liquid Logic, Cubist, Cerexa, MedImmune, Merck, NIH, Novartis, Pfizer, and Theravance; has served as paid consultant for Affinium, Baxter, Cerexa, Cubist, Debiopharm, Durata, Merck, Novartis, NovaDigm, The Medicines Company, MedImmune, Pfizer, Theravance, and Trius; has received honoraria from Arpida, Astellas, Cubist, Inhibitex, Merck, Pfizer, Targanta, Theravance, Wyeth, Ortho-McNeil, Novartis, and Vertex Pharmaceuticals; and has membership as Merck Co-Chair V710 Vaccine.
D.V.D. reports that he is on the advisory board of Actavis, Tetraphase, and Sanofi-Pasteur and has received research funding from Steris and from Scynexis. All other authors report no conflicts relevant to this article.

Address correspondence to David van Duin, $\mathrm{MD}, \mathrm{PhD}$, Division of Infectious Diseases, CB 7030, University of North Carolina, 130 Mason Farm Rd, Chapel Hill, NC 27599 (david_vanduin@med.unc.edu).

\section{REFERENCES}

1. Borer A, Saidel-Odes L, Riesenberg K, et al. Attributable mortality rate for carbapenem-resistant Klebsiella pneumoniae bacteremia. Infect Control Hosp Epidemiol 2009;30:972-976.

2. Nguyen M, Eschenauer GA, Bryan M, et al. Carbapenemresistant Klebsiella pneumoniae bacteremia: factors correlated with clinical and microbiologic outcomes. Diagn Microbiol Infect Dis 2010;67:180-184.

3. Munoz-Price LS, Poirel L, Bonomo RA, et al. Clinical epidemiology of the global expansion of Klebsiella pneumoniae carbapenemases. Lancet Infect Dis 2013;13:785-796.

4. Centers for Disease Control and Prevention. Vital signs: carbapenem-resistant Enterobacteriaceae. MMWR Morb Mortal Wkly Rep 2013;62:165-170.

5. Thaden JT, Lewis SS, Hazen KC, et al. Rising rates of carbapenemresistant Enterobacteriaceae in community hospitals: a mixedmethods review of epidemiology and microbiology practices in a network of community hospitals in the southeastern United States. Infect Control Hosp Epidemiol 2014;35:978-983.

6. Arnold RS, Thom KA, Sharma S, Phillips M, Kristie Johnson J, Morgan DJ. Emergence of Klebsiella pneumoniae carbapenemaseproducing bacteria. South Med J 2011;104:40-45.

7. Benenson S, Warburg G, Hidalgo-Grass C, et al. Comparison of two carbapenem-resistant Klebsiella pneumoniae clones: from a contained outbreak in a paediatric population and from a national epidemic. J Antimicrob Chemother 2012;67:1651-1654.

8. Bratu S, Landman D, Haag R, et al. Rapid spread of carbapenemresistant Klebsiella pneumoniae in New York City: a new threat to our antibiotic armamentarium. Arch Intern Med 2005;165:1430-1435.

9. Leavitt A, Navon-Venezia S, Chmelnitsky I, Schwaber MJ, Carmeli Y. Emergence of KPC-2 and KPC-3 in carbapenemresistant Klebsiella pneumoniae strains in an Israeli hospital. Antimicrob Agents Chemother 2007;51:3026-3029.

10. Prabaker K, Lin MY, McNally M, et al. Transfer from high-acuity long-term care facilities is associated with carriage of Klebsiella pneumoniae carbapenemase-producing Enterobacteriaceae: a multihospital study. Infect Control Hosp Epidemiol 2012;33:1193-1199.

11. van Duin D, Perez F, Rudin SD, et al. Surveillance of carbapenem-resistant Klebsiella pneumoniae: tracking molecular epidemiology and outcomes through a regional network. Antimicrob Agents Chemother 2014;58:4035-4041.

12. van Duin D, Cober E, Richter SS, et al. Impact of therapy and strain type on outcomes in urinary tract infections caused by carbapenem-resistant Klebsiella pneumoniae. J Antimicrob Chemother 2015;70:1203-1211.

13. Chow JW, Yu VL. Combination antibiotic therapy versus monotherapy for gram-negative bacteraemia: a commentary. Int J Antimicrob Agents 1999;11:7-12.

14. Charlson ME, Pompei P, Ales KL, MacKenzie CR. A new method of classifying prognostic comorbidity in longitudinal studies: development and validation. J Chronic Dis 1987;40:373-383. 
15. Clinical and Laboratory Standards Institute. Performance standards for antimicrobial susceptibility testing: 24th informational supplement. CLSI document. Wayne, PA: CLSI, 2014:M100-S24.

16. Kansagara D, Englander H, Salanitro A, et al. Risk prediction models for hospital readmission: a systematic review. JAMA 2011;306:1688-1698.

17. Hemkens LG, Contopoulos-Ioannidis DG, Ioannidis JP. Concordance of effects of medical interventions on hospital admission and readmission rates with effects on mortality. CMAJ 2013;185:E827-E837.

18. Prescott HC, Langa KM, Iwashyna TJ. Readmission diagnoses after hospitalization for severe sepsis and other acute medical conditions. JAMA 2015;313:1055-1057.

19. Zimmerman FS, Assous MV, Bdolah-Abram T, Lachish T, Yinnon AM, Wiener-Well Y. Duration of carriage of carbapenem-resistant Enterobacteriaceae following hospital discharge. Am J Infect Control 2013;41:190-194.

20. Schechner V, Kotlovsky T, Tarabeia J, et al. Predictors of rectal carriage of carbapenem-resistant Enterobacteriaceae (CRE) among patients with known CRE carriage at their next hospital encounter. Infect Control Hosp Epidemiol 2011;32:497-503.

21. Bart Y, Paul M, Eluk O, Geffen Y, Rabino G, Hussein K. Risk factors for recurrence of carbapenem-resistant Enterobacteriaceae carriage: case-control study. Infect Control Hosp Epidemiol 2015;36:936-941.

22. Perez F, Pultz MJ, Endimiani A, Bonomo RA, Donskey CJ. Effect of antibiotic treatment on establishment and elimination of intestinal colonization by KPC-producing Klebsiella pneumoniae in mice. Antimicrob Agents Chemother 2011;55:2585-2589.

23. Saidel-Odes L, Polachek $\mathrm{H}$, Peled $\mathrm{N}$, et al. A randomized, double-blind, placebo-controlled trial of selective digestive decontamination using oral gentamicin and oral polymyxin $\mathrm{E}$ for eradication of carbapenem-resistant Klebsiella pneumoniae carriage. Infect Control Hosp Epidemiol 2012;33:14-19.
24. Garrett WS. Cancer and the microbiota. Science 2015;348: 80-86.

25. Tasina E, Haidich AB, Kokkali S, Arvanitidou M. Efficacy and safety of tigecycline for the treatment of infectious diseases: a meta-analysis. Lancet Infect Dis 2011;11:834-844.

26. Cai Y, Wang R, Liang B, Bai N, Liu Y. Systematic review and meta-analysis of the effectiveness and safety of tigecycline for treatment of infectious disease. Antimicrob Agents Chemother 2011;55:1162-1172.

27. Prasad P, Sun J, Danner RL, Natanson C. Excess deaths associated with tigecycline after approval based on noninferiority trials. Clin Infect Dis 2012;54:1699-1709.

28. Yahav D, Lador A, Paul M, Leibovici L. Efficacy and safety of tigecycline: a systematic review and meta-analysis. J Antimicrob Chemother 2011;66:1963-1971.

29. Xie J, Wang T, Sun J, et al. Optimal tigecycline dosage regimen is urgently needed: results from a pharmacokinetic/pharmacodynamic analysis of tigecycline by Monte Carlo simulation. Int $J$ Infect Dis 2014;18:62-67.

30. Schwaber MJ, Lev B, Israeli A, et al. Containment of a countrywide outbreak of carbapenem-resistant Klebsiella pneumoniae in Israeli hospitals via a nationally implemented intervention. Clin Infect Dis 2011;52:848-855.

31. Ben-David D, Maor Y, Keller N, et al. Potential role of active surveillance in the control of a hospital-wide outbreak of carbapenem-resistant Klebsiella pneumoniae infection. Infect Control Hosp Epidemiol 2010;31:620-626.

32. Munoz-Price LS, Hayden MK, Lolans K, et al. Successful control of an outbreak of Klebsiella pneumoniae carbapenemase-producing K. pneumoniae at a long-term acute care hospital. Infect Control Hosp Epidemiol 2010;31:341-347.

33. Kochar S, Sheard T, Sharma R, et al. Success of an infection control program to reduce the spread of carbapenem-resistant Klebsiella pneumoniae. Infect Control Hosp Epidemiol 2009;30:447-452. 